\title{
Factors Associated to Online Shopping at the BoP Community in Rural Bangladesh
}

\author{
Kazi Mozaher Hossein \\ Department of Advanced \\ Information Technology \\ Kyushu University, \\ Fukuoka, Japan
}

Fumihiko Yokota

Institute of Decision Science for a

Sustainable Society

Kyushu University,

Fukuoka, Japan

\author{
Mariko Nishikitani \\ Institute of Decision Science for a \\ Sustainable Society \\ Kyushu University, \\ Fukuoka, Japan \\ Rafiqul Islam \\ Global Communication Center \\ Grameen Communications, \\ Dhaka, Bangladesh
}

\author{
Hironobu Kitaoka \\ Toyota Motor Corporation \\ Aichi, \\ Japan \\ Hiroshi Okajima \\ Toyota Motor Corporation \\ Aichi, \\ Japan
}

\author{
Akira Fukuda \\ Department of Advanced Information Technology \\ Kyushu University, \\ Fukuoka, Japan
}

\author{
Ashir Ahmed \\ Department of Advanced Information Technology \\ Kyushu University, \\ Fukuoka, Japan
}

\begin{abstract}
Online shopping is getting popular even in the rural areas of developing countries. However, few research has been conducted to identify the factors associated to online shopping by the poor villagers. Whereas people living at the bottom of the economic pyramid (BoP) has an aggregate purchase power which is a huge market and online shopping has the potentiality in reducing $\mathrm{BoP}$ penalty by removing unnecessary middlemen from the supply chain. In this research, we have conducted a field survey on 600 households in the western part of rural Bangladesh to find out current status of online shopping use by the BoP people and the demographic and behavioral factors associated with online shopping. Chi-square test of association and multi-variate logistic regression test have been performed to analyze data. Result shows that cell phone use, computer use, social media use, and mobile money transfer use have significant relationship in online shopping use at the BoP community.
\end{abstract}

Keywords-Online shopping; BoP; demographic and behavioral factors; Bangladesh

\section{INTRODUCTION}

Online shopping has been gaining acceptance rapidly due to the dot-com boom in the 90s globally [1]. The level of its acceptance and popularity has been growing since it has the potentiality to go beyond international boundaries enabling activities within the virtual marketplace. It has the flexibility of time and distance [2] in purchasing products. It provides wider product selection opportunity within limited space. Overall, it saves time, money and labor [3] of the consumers. Several studies on the usage of online shopping identified different factors, such as demographic factors, behavioral factors, infrastructural factors and so on [4]-[6]. Different studies identified the barriers of online shopping expansion globally
[7], [8]. If we see the basic requirement of online shopping usage we find there are three major components to access online shopping such as; 1) Internet connectivity to access online catalog, 2) Payment mechanism such as credit card, mobile wallet etc. to make payment, and 3) Product delivery infrastructure [3].

However, these components are not equally ready everywhere in the world so that the consumers could get equal benefit from online shopping. Online shopping service primarily designed targeting the consumers at the top of the economic pyramid and gradually expanding towards the middle of the economic pyramid. Bottom of the economic pyramid (BoP) had been ignored. Currently, there are 4 billion people at the $\mathrm{BoP}$, comprising $54 \%$ of the world population [9], [10]. Disposable income of the people in this segment is basically low [11] and shopping for daily needs is the norm and online shopping is not a priority. However, this scenario will change as more consumers continue to move up the socioeconomic ladder.

Bangladesh, a developing country where online shopping usage continues at a rapid pace with recent growth of Internet usage, 3G roll-out in 2013 [12]. Mobile money transfer service replaced the need of credit or debit card as payment mechanism. Different product delivery companies are starting up to fulfill the need of online shopping product delivery. This infrastructural environment is increasing the use of online shopping and currently it is much popular in major urban areas in the country [13]. Although online shopping usage at the rural areas in Bangladesh is limited compared to the urban areas but it is expected to increase in the long run as we can see online shopping started to become popular in the rural areas of developing countries such as China and India [14]. Several 
researches have been conducted to identify the factors of online shopping. However, few research has been conducted to identify the factors associated to online shopping targeting the BoP community. This research is much important to the online shopping service providers so that they can design the service more efficiently after knowing the factors those affected behind online shopping decision making by the current online shopping users at the BoP community. Therefore, this research attempts to identify the factors that are primarily responsible behind online shopping by the BoP consumers. It aims to find the differences in socio-demographic and behavioral characteristics between low and high family expenditure groups of the online shopping users in BoP community.

Section II explains the motivation behind this research and Section III introduces the method of the experiment. Section IV describes the findings, Section $\mathrm{V}$ discusses the findings and Section VI concludes the article.

\section{RESEARCH MOTIVATION}

This research started with a strong motivation to explore the existence and reasons of BoP penalty, and to design ICT based social services those can reduce the BoP penalty. The people at the BoP often pay higher prices for basic goods and services than do wealthier consumers [9]. Toyota Motor Corporation and Kyushu University in Japan are jointly carrying out a research and implemented in Bangladesh to see whether ICT and mobility could reduce BoP penalty or not since 2011. Grameen Communications in Bangladesh is supporting the experiment. A 10 seated Toyota Hiace vehicle is shared to carry ICT based 4 major services such as: 1) Remote healthcare; 2) Female friendly transport using car sharing model; 3) E-learning; and 4) Last mile delivery service of online purchase. This research project is known as Social Services on Wheels (SSW) [15]. Under SSW project there were two experimental sites in two districts in Bangladesh. One was in Kalihati Upazila under Tangail district $(105 \mathrm{~km}$ away from the capital Dhaka city) and the other one was in Bheramara Upazila under Kushtia district (235 km away from the capital Dhaka city). In this research, we have used Bheramara Upazila as experimental platform. Bheramara is located in the western part of Bangladesh under Kushtia district and is close to the border of India. According to the census of Bangladesh Bureau of Statistics (BBS) 2011, the Bheramara sub-district has a population of 208,000 in six unions. We have found the existence of BoP penalty (Table I) from a baseline survey conducted in a rural area (Bheramara Upazila) and an urban area (Dhaka city). However, we argue that, online shopping can be used to reduce BoP penalty [3] as it has the potentiality to enable villagers to purch ase products from a village at any time of the day and get the desired products delivered to their doors. In this research we planned to explore current online shopping user status, online shopping use environment, and the factors that affected online shopping decision making in our experimental site.
TABLE I. PRACTICAL EXAMPLES OF BOP PENALTY

\begin{tabular}{|l|l|l|l|l|}
\hline \multicolumn{1}{|c|}{ Item } & $\begin{array}{l}\text { Bheramara } \\
\text { [Taka] }\end{array}$ & $\begin{array}{l}\text { Dhaka } \\
{[\text { Taka }]}\end{array}$ & $\begin{array}{l}\text { Poverty } \\
\text { Penalty } \\
{[\%]}\end{array}$ & Category \\
\hline Mineral water (500 ml) & 18 & 15 & 20 & $\begin{array}{l}\text { Food and } \\
\text { Nutrition }\end{array}$ \\
\hline Blood glucose test (1 time) & 300 & 150 & 100 & Healthcare \\
\hline Rice (1 kg) & 60 & 50 & 20 & $\begin{array}{l}\text { Food and } \\
\text { Nutrition }\end{array}$ \\
\hline LP gas cylinder (12 kg) & 1000 & 800 & 25 & $\begin{array}{l}\text { Home } \\
\text { appliance }\end{array}$ \\
\hline Mosquito net (1 piece) & 950 & 800 & 18.75 & Healthcare \\
\hline T-shirt (1 piece) & 400 & 300 & 33.33 & Clothing \\
\hline Rickshaw fare (1 km) & 15 & 20 & $(25)$ & Mobility \\
\hline Paracetamol (1 tablet) & 2 & 2 & 0 & Healthcare \\
\hline Soap (1 piece) & 20 & 20 & 0 & FMCG \\
\hline
\end{tabular}

\section{METHODOLOGY}

\section{A. Data Source and Data Collection Procedures}

We have conducted a field survey at one of the experimental sites of SSW project to collect data from community residents of Bheramara sub-district to identify online shopping user status, online shopping use environment, and the factors that affected behind online shopping decision making. The survey profile is mentioned in Table II below.

TABLE II. SURVEY PROFILE

\begin{tabular}{|l|l|}
\hline Location & $\begin{array}{l}\text { Bheramara sub-district, Kushtia district, } \\
\text { Bangladesh }\end{array}$ \\
\hline Duration & June 1 to June 15, 2016 \\
\hline Mode of interview & $\begin{array}{l}\text { Face-to-face interview using semi-structured } \\
\text { questionnaire }\end{array}$ \\
\hline Type of sampling & Stratified random sampling \\
\hline Sample size & 600 households \\
\hline $\begin{array}{l}\text { Area covered during } \\
\text { the survey }\end{array}$ & $\begin{array}{l}6 \text { unions of Bheramara sub-district. 100 samples } \\
\text { from each union }\end{array}$ \\
\hline
\end{tabular}


Participants were individuals from the rural households Only one respondent was interviewed from one household. Three field staffs who had been working with SSW project were trained with a semi-structured questionnaire to collect data from the rural households.

\section{B. Dependent and Independent Variables and Measurements}

The outcome variable was online shopping use. Online shopping use was defined as online shopping experience by the respondents once in their lifetime and was classified dichotomously as "yes" or "no". All the independent variables (computer use, social media use, and mobile money transfer use) except cell phone user per family were dichotomously coded as "yes" or "no". Cell phone user per family was coded as "two or less $(n \leq 2)$ " and "three or more $(n \geq 3)$ " per family. Age, gender, employment status, education, and monthly family expenditure were selected as the main control variables. The households who had monthly family expenditure of 10000 taka or less belonged to low family expenditure group and the households who had more than 10000 taka as monthly family expenditure belonged to high family expenditure group.

\section{Data Analysis}

Socio-demographic and behavioral characteristics of the sample were analyzed separately by low family and high family expenditure groups (Table III). Pearson's chi-square test of association was performed to describe the unadjusted association between dependent and independent/control categorical variables (Table IV). Multiple logistic regression analysis was performed to describe the adjusted association of independent variables with the outcome variable online shopping use after adjusting with age, gender, employment status, education, and monthly family expenditure (Table V). Odds ratio (OR) and 95\% confidence interval (95\% CI) were calculated. Each independent variable was included in the logistic regression models separately because the independent variables were highly correlated (multi-collinearity). Thus, the following four distinct models were used considering one independent variable in one model:

Model 1: Logit Y1 $(\mathrm{X})=\beta 0+\beta 1($ age $)+\beta 2($ gender $)+\beta 3$ (employment status) $+\beta 4$ (education) $+\beta 5$ (cell phone user per family) $+\varepsilon$

Model 2: Logit Y1 $(\mathrm{X})=\beta 0+\beta 1$ (age) $+\beta 2$ (gender) $+\beta 3$ (employment status) $+\beta 4$ (education) $+\beta 5$ (computer use $)+\varepsilon$

Model 3: Logit Y1 $(\mathrm{X})=\beta 0+\beta 1$ (age) $+\beta 2$ (gender) $+\beta 3$ (employment status) $+\beta 4$ (education) $+\beta 5$ (social media use) $+\varepsilon$

Model 4: Logit Y1 $(\mathrm{X})=\beta 0+\beta 1$ (age) $+\beta 2$ (gender) $+\beta 3$ (employment status) $+\beta 4$ (education) $+\beta 5$ (mobile money transfer use) $+\varepsilon$

All statistical analyses were performed using SPSS version 22 (IBM Corp., Armonk, NY). A P-value <0.05 was considered significant.

\section{FINDINGS}

Age range of the samples was from 15 to 80 years, and mean age was 37 (Table III). More than half (54\%) of the samples were within the age-group of 15-39. Majority (79\%) of the samples were male. A total of $35 \%$ of the samples were unemployed [ $\mathrm{N}=558]$. However, students and housewives were the major portion of the unemployed samples. Majority $(86 \%)$ of the samples had high school level or less (up to grade 12) education [ $\mathrm{N}=539]$. $14 \%$ of the samples had higher education. In low family expenditure group, $7 \%$ people had higher education where it was $18 \%$ in high family expenditure group. $34 \%$ households had 3 or more cellphones. In high family expenditure group having 3 or more cellphones was more than double (43\%) compared to the low family expenditure group (20\%). $28 \%$ of the total samples reported as computer user which is almost $20 \%$ for low family expenditure group and $33 \%$ for high family expenditure group. One fifth $(20 \%)$ of the respondents were social media user, $14 \%$ belonged to low family and $23 \%$ belonged to high family expenditure group. More than half $(54 \%)$ of the respondents were mobile money transfer service user, $61 \%$ belonged to high family expenditure and $44 \%$ belonged to low family expenditure group. More than one-fifth $(22 \%)$ of the respondents were known to online shopping, however, less than half of the respondents in low family expenditure group (12\%) were known to online shopping compared to high family expenditure group (29\%). Out of 587 respondents, $51(9 \%)$ used online shopping. In low family expenditure group $6 \%$ respondents used online shopping and in high family expenditure group $11 \%$ respondents used online shopping.

Education showed a significant association (Table IV) with online shopping usage $(\mathrm{P}<0.001)$ in total and high family expenditure groups. However, in low family expenditure group the relationship was not significant $(\mathrm{P}=0.214) .21 .1 \%$ of the respondent who had higher education used online shopping. On the other hand, $6.7 \%$ respondents who had up to 12 years of education used online shopping. Monthly family expenditure had a significant association $(\mathrm{P}<0.05)$ with online shopping usage. $5.9 \%$ respondents who had 10000 taka or less as monthly family expenditure used online shopping. Compared to that, almost double (11\%) respondents used online shopping who had monthly family expenditure more than 10000 taka. Use of computer $(\mathrm{P}<0.001)$ had a significant association with online shopping usage. There was a significant relationship $(\mathrm{P}=0.024)$ between online shopping use and cell phone user per family. $16.2 \%$ respondents who had 3 or more cell phone users in their households used online shopping compared to $9.0 \%$ respondents who had 2 or less cell phone user. $22.2 \%$ of the computer user was online shopping user as well. More than double respondents used online shopping who belonged to high family expenditure computer user group (26.1\%) compared to low family expenditure computer user group (12.8\%). Use of social media $(\mathrm{P}<0.001)$ had a significant association with online shopping usage. $20.9 \%$ of the respondents used social media who were also online shopping user. More than double respondents used online shopping who belonged to high family expenditure social media user group (24.7\%) compared to low family expenditure social media user group (11.8\%). Mobile money transfer user $(\mathrm{P}<0.01)$ showed a significant association with online shopping usage. However, a border line association was found for low family expenditure group $(\mathrm{P}=0.091) .11 .7 \%$ of mobile money transfer user used online shopping. Knowing online shopping showed a significant association with online shopping $(\mathrm{P}<0.01)$. However, for low and high family 
expenditure groups online shopping user percentage did not differ significantly ( $27.6 \%$ and $32.0 \%$ respectively).

TABLE III. SOCIO-DEMOGRAPHIC AND BEHAVIORAL CHARACTERISTICS OF THE SURVEYED SAMPLES COMPARING HIGH AND LOW FAMILY EXPENDITURE GROUPS

\begin{tabular}{|c|c|c|c|c|c|c|}
\hline \multirow[t]{2}{*}{ Items } & \multicolumn{2}{|l|}{ Total } & \multicolumn{2}{|c|}{\begin{tabular}{|l} 
Low family \\
expenditure \\
$(\leq 10000$ taka)
\end{tabular}} & \multicolumn{2}{|c|}{$\begin{array}{l}\text { High family } \\
\text { expenditure } \\
(>10000 \text { taka) }\end{array}$} \\
\hline & $N$ & $\%$ & $N$ & $\%$ & $N$ & $\%$ \\
\hline Age group & $N=587$ & $\begin{array}{l}\text { Mean = } \\
36.82 \\
\text { Range = } \\
15-80\end{array}$ & $N=239$ & \begin{tabular}{|l} 
Mean $=$ \\
33.21 \\
Range $=$ \\
$15-73$
\end{tabular} & $\begin{array}{l}N= \\
348\end{array}$ & $\begin{array}{l}\text { Mean = } \\
39.29 \\
\text { Range }= \\
15-80\end{array}$ \\
\hline $15-29$ years & 198 & 34.0 & 83 & 34.7 & 90 & 25.9 \\
\hline $30-39$ years & 117 & 20.0 & 76 & 31.8 & 66 & 19.0 \\
\hline $40-49$ years & 141 & 24.0 & 50 & 20.9 & 91 & 26.1 \\
\hline $50-59$ years & 86 & 15.0 & 22 & 9.2 & 64 & 18.4 \\
\hline$\geq 60$ years & 45 & 7.0 & 8 & 3.3 & 37 & 10.6 \\
\hline Gender & $\mathrm{N}=587$ & & $\mathrm{~N}=239$ & & $\mathrm{~N}=348$ & \\
\hline Male & 465 & 79.0 & 177 & 74.1 & 288 & 82.8 \\
\hline Female & 122 & 21.0 & 62 & 25.9 & 60 & 17.2 \\
\hline $\begin{array}{l}\text { Employment } \\
\text { status }\end{array}$ & $\mathrm{N}=558$ & & $\mathrm{~N}=230$ & & $\mathrm{~N}=328$ & \\
\hline Unemployed & 197 & 35.0 & 96 & 41.8 & 101 & 30.8 \\
\hline Employed & 361 & 65.0 & 134 & 58.2 & 227 & 69.2 \\
\hline Education & $\mathrm{N}=539$ & & $\mathrm{~N}=206$ & & $\mathrm{~N}=333$ & \\
\hline$\leq$ Grade 12 & 463 & 85.9 & 191 & 92.7 & 272 & 81.7 \\
\hline$>$ Grade 12 & 76 & 14.1 & 15 & 7.3 & 61 & 18.3 \\
\hline $\begin{array}{l}\text { Cell phone user } \\
\text { per family }\end{array}$ & $\mathrm{N}=587$ & & $\mathrm{~N}=239$ & & $\mathrm{~N}=348$ & \\
\hline $\mathrm{n} \leq 2$ & 389 & 66.3 & 192 & 80.3 & 197 & 56.6 \\
\hline $\mathrm{n} \geq 3$ & 198 & 33.7 & 47 & 19.7 & 151 & 43.4 \\
\hline Computer use & $\mathrm{N}=587$ & & $\mathrm{~N}=239$ & & $\mathrm{~N}=348$ & \\
\hline Yes & 162 & 28.0 & \begin{tabular}{|l|l|}
47 \\
\end{tabular} & 19.7 & 115 & 33.0 \\
\hline No & 425 & 72.0 & 192 & 80.3 & 233 & 67.0 \\
\hline Social media use & $\mathrm{N}=587$ & & $\mathrm{~N}=239$ & & $\mathrm{~N}=348$ & \\
\hline Yes & 115 & 20.0 & \begin{tabular}{|l|}
34 \\
\end{tabular} & 14.2 & 81 & 23.3 \\
\hline No & 472 & 80.0 & 205 & 85.8 & 267 & 76.7 \\
\hline $\begin{array}{l}\text { Mobile money } \\
\text { transfer use }\end{array}$ & $\mathrm{N}=587$ & & $\mathrm{~N}=239$ & & $\mathrm{~N}=348$ & \\
\hline Yes & 316 & 54.0 & 104 & 43.5 & 212 & 60.9 \\
\hline No & 271 & 46.0 & 135 & 56.5 & 136 & 39.1 \\
\hline $\begin{array}{l}\text { Knowing online } \\
\text { shopping }\end{array}$ & $\mathrm{N}=587$ & & $\mathrm{~N}=239$ & & $\mathrm{~N}=348$ & \\
\hline Yes & 129 & 22.0 & 29 & 12.1 & 100 & 28.7 \\
\hline No & 458 & 78.0 & 210 & 87.9 & 248 & 71.3 \\
\hline $\begin{array}{l}\text { Online } \\
\text { shopping use }\end{array}$ & $\mathrm{N}=587$ & & $\mathrm{~N}=239$ & & $\mathrm{~N}=348$ & \\
\hline Yes & 51 & 9.0 & 14 & 5.9 & 37 & 10.6 \\
\hline No & 536 & 91.0 & 225 & 94.1 & 311 & 89.4 \\
\hline
\end{tabular}

TABLE IV. CHI-SQUARE TEST RESULTS OF DEPENDENT AND INDEPENDENT VARIABLES AND A COMPARISON BETWEEN HIGH AND LOW FAMILY EXPENDITURE GROUPS

\begin{tabular}{|c|c|c|c|c|c|c|c|c|c|c|c|c|}
\hline \multicolumn{13}{|c|}{ Online shopping user (Yes, $\mathbf{n = 5 1}$ ) } \\
\hline \multirow[b]{2}{*}{$\begin{array}{l}\text { Characteristi } \\
\text { cs }\end{array}$} & \multicolumn{4}{|c|}{ Total } & \multicolumn{4}{|c|}{$\begin{array}{l}\text { Low family } \\
\text { expenditure } \\
(\leq 10000 \text { taka })\end{array}$} & \multicolumn{4}{|c|}{$\begin{array}{l}\text { High family } \\
\text { expenditure } \\
(>10000 \text { taka) }\end{array}$} \\
\hline & $N$ & $n$ & $\%$ & \begin{tabular}{|l|}
$p-$ \\
value
\end{tabular} & $N$ & $n$ & $\%$ & \begin{tabular}{|l}
$p-$ \\
valu \\
$e$
\end{tabular} & $N$ & $n$ & $\%$ & $\begin{array}{l}p \text { - } \\
\text { value }\end{array}$ \\
\hline Age group & $\begin{array}{l}N=58 \\
7\end{array}$ & $\begin{array}{l}n=5 \\
1\end{array}$ & & 0.173 & $\begin{array}{l}N=23 \\
9\end{array}$ & $\begin{array}{l}n=1 \\
4\end{array}$ & & $\begin{array}{l}0.06 \\
7\end{array}$ & $\begin{array}{l}N=34 \\
8\end{array}$ & $\begin{array}{l}n=3 \\
7\end{array}$ & & 0.221 \\
\hline $15-29$ & 220 & 24 & $\begin{array}{l}10 . \\
9\end{array}$ & & 99 & 9 & 9.1 & & 99 & 13 & $\begin{array}{l}13 . \\
1\end{array}$ & \\
\hline$\geq 30$ & 367 & 27 & 7.4 & & 140 & 5 & 3.6 & & 249 & 24 & 9.6 & \\
\hline Gender & $\begin{array}{l}\mathrm{N}=58 \\
7\end{array}$ & $\begin{array}{l}n=5 \\
1\end{array}$ & & 0.277 & $\begin{array}{l}\mathrm{N}=23 \\
9\end{array}$ & $\begin{array}{l}n=1 \\
4\end{array}$ & & $\begin{array}{l}0.51 \\
4\end{array}$ & 348 & 37 & & 0.086 \\
\hline Male & 465 & 44 & 9.5 & & 177 & 10 & 5.6 & & 288 & 34 & $\begin{array}{l}11 . \\
8\end{array}$ & \\
\hline Female & 122 & 7 & 5.7 & & 62 & 4 & 6.5 & & 60 & 3 & 5.0 & \\
\hline $\begin{array}{l}\text { Employment } \\
\text { status }\end{array}$ & $\begin{array}{l}N=55 \\
8\end{array}$ & $\begin{array}{l}n=4 \\
9\end{array}$ & & 0.350 & $\begin{array}{l}\mathrm{N}=23 \\
0\end{array}$ & $\begin{array}{l}n=1 \\
3\end{array}$ & & $\begin{array}{l}0.75 \\
0\end{array}$ & $\begin{array}{l}\mathrm{N}=32 \\
8\end{array}$ & $\begin{array}{l}n=3 \\
6\end{array}$ & & 0.343 \\
\hline Unemployed & 197 & 14 & 7.1 & & 96 & 6 & 6.3 & & 101 & 8 & 7.9 & \\
\hline Employed & 361 & 35 & 9.7 & & 134 & 7 & 5.2 & & 227 & 28 & $\begin{array}{l}12 . \\
3\end{array}$ & \\
\hline Education & $\begin{array}{l}N=53 \\
9\end{array}$ & $\begin{array}{l}n=4 \\
7\end{array}$ & & $\begin{array}{l}<0.00 \\
1\end{array}$ & $\begin{array}{l}N=20 \\
6\end{array}$ & $\begin{array}{l}n=1 \\
2\end{array}$ & & $\begin{array}{l}0.21 \\
4\end{array}$ & $\begin{array}{l}N=33 \\
3\end{array}$ & $\begin{array}{l}n=3 \\
5\end{array}$ & & 0.001 \\
\hline$\leq$ Grade 12 & 463 & 31 & 6.7 & & 191 & 10 & 5.2 & & 272 & 21 & 7.7 & \\
\hline$>$ Grade 12 & 76 & 16 & $\begin{array}{l}21 . \\
1\end{array}$ & & 15 & 2 & $\begin{array}{l}13 . \\
3\end{array}$ & & 61 & 14 & $\begin{array}{l}23 . \\
0\end{array}$ & \\
\hline \begin{tabular}{|l|l}
$\begin{array}{l}\text { Monthly } \\
\text { family } \\
\text { expenditure }\end{array}$ \\
\end{tabular} & $\begin{array}{l}N=57 \\
5\end{array}$ & $\begin{array}{l}n=5 \\
1\end{array}$ & & $<0.05$ & & & & & & & & \\
\hline$\leq 10000$ & 239 & 14 & 5.9 & & & & & & & & & \\
\hline$>10000$ & 336 & 37 & 11. & & & & & & & & & \\
\hline \begin{tabular}{|l|} 
Cell phone \\
user per \\
family
\end{tabular} & $\begin{array}{l}\mathrm{N}=58 \\
7\end{array}$ & $\begin{array}{l}n=5 \\
1\end{array}$ & & 0.024 & $\begin{array}{l}\mathrm{N}=23 \\
9\end{array}$ & $\begin{array}{l}n=1 \\
4\end{array}$ & & $\begin{array}{l}0.03 \\
6\end{array}$ & $\begin{array}{l}N=34 \\
8\end{array}$ & $\begin{array}{l}n=3 \\
7\end{array}$ & & $\begin{array}{l}<0.00 \\
1\end{array}$ \\
\hline $\mathrm{n} \leq 2$ & 389 & 19 & 9.0 & & 192 & 8 & 4.2 & & 197 & 11 & 5.6 & \\
\hline$n \geq 3$ & 198 & 32 & 16. & & 47 & 6 & 12. & & 151 & 26 & $\begin{array}{l}17 . \\
2\end{array}$ & \\
\hline $\begin{array}{l}\text { Computer } \\
\text { use }\end{array}$ & $\begin{array}{l}N=58 \\
7\end{array}$ & $\begin{array}{l}n=5 \\
1\end{array}$ & & \begin{tabular}{|l|}
$<0.00$ \\
1
\end{tabular} & $\begin{array}{l}N=23 \\
9\end{array}$ & $\begin{array}{l}n=1 \\
4\end{array}$ & & \begin{tabular}{|l|}
0.03 \\
6
\end{tabular} & $\begin{array}{l}N=34 \\
8\end{array}$ & $\begin{array}{l}n=3 \\
7\end{array}$ & & $\begin{array}{l}<0.00 \\
1\end{array}$ \\
\hline Yes & 162 & 36 & 22. & & 47 & 6 & $\begin{array}{l}12 . \\
8\end{array}$ & & 115 & 30 & $\begin{array}{l}26 . \\
1\end{array}$ & \\
\hline No & 425 & 15 & 3.5 & & 192 & 8 & 4.2 & & 233 & 7 & 3.0 & \\
\hline $\begin{array}{l}\text { Social media } \\
\text { use }\end{array}$ & $\begin{array}{l}N=58 \\
7\end{array}$ & $\begin{array}{l}n=5 \\
1\end{array}$ & & \begin{tabular}{|l|}
$<0.00$ \\
1 \\
\end{tabular} & $\begin{array}{l}\mathrm{N}=23 \\
9\end{array}$ & $\begin{array}{l}n=1 \\
4\end{array}$ & & \begin{tabular}{|l|}
0.12 \\
0
\end{tabular} & $\begin{array}{l}N=34 \\
8 \\
\end{array}$ & $\begin{array}{l}n=3 \\
7\end{array}$ & & $\begin{array}{l}<0.00 \\
1 \\
\end{array}$ \\
\hline Yes & 115 & 24 & $\begin{array}{l}20 . \\
9\end{array}$ & & 34 & 4 & $\begin{array}{l}11 . \\
8\end{array}$ & & 81 & 20 & 24. & \\
\hline No & 472 & 27 & 5.7 & & 205 & 10 & 4.9 & & 267 & 17 & 6.4 & \\
\hline $\begin{array}{l}\text { Mobile } \\
\text { money } \\
\text { transfer use }\end{array}$ & $\begin{array}{l}\mathrm{N}=58 \\
7\end{array}$ & $\begin{array}{l}n=5 \\
1\end{array}$ & & $<0.01$ & $\begin{array}{l}\mathrm{N}=23 \\
9\end{array}$ & $\begin{array}{l}n=1 \\
4\end{array}$ & & $\begin{array}{l}0.09 \\
1\end{array}$ & $\begin{array}{l}\mathrm{N}=34 \\
8\end{array}$ & $\begin{array}{l}n=3 \\
7\end{array}$ & & 0.036 \\
\hline Yes & 316 & 37 & 11. & & 104 & 9 & 8.7 & & 212 & 28 & $\begin{array}{l}13 . \\
2\end{array}$ & \\
\hline No & 271 & 14 & 5.2 & & 135 & 5 & 3.7 & & 136 & 9 & 6.6 & \\
\hline $\begin{array}{l}\begin{array}{l}\text { Knowing } \\
\text { online } \\
\text { shopping }\end{array} \\
\end{array}$ & $\begin{array}{l}\mathrm{N}=58 \\
7\end{array}$ & $\begin{array}{l}n=5 \\
1\end{array}$ & & $<0.01$ & $\begin{array}{l}\mathrm{N}=23 \\
9\end{array}$ & $\begin{array}{l}n=1 \\
4\end{array}$ & & $\begin{array}{l}<0.0 \\
1\end{array}$ & $\begin{array}{l}N=34 \\
8\end{array}$ & $\begin{array}{l}n=3 \\
7\end{array}$ & & $<0.01$ \\
\hline Yes & 129 & 40 & $\begin{array}{l}31 . \\
0\end{array}$ & & 29 & 8 & $\begin{array}{l}27 . \\
6\end{array}$ & & 100 & 32 & $\begin{array}{l}32 . \\
0\end{array}$ & \\
\hline No & 458 & 11 & 2.4 & & 210 & 6 & 2.9 & & 248 & 5 & 2.0 & \\
\hline
\end{tabular}


Table V displays the results of Model 1 and 2 and Table VI displays the results of Model 3 and 4 of multivariate logistic regression analysis. Model 1 shows that participants who had 3 or more cell phone users in their households were 3.4 times more likely to use online shopping compared to the participants who had 2 or less cell phone users $(\mathrm{OR}=3.41, \mathrm{P}<0.001)$ after adjusting age, gender, employment status, education, and monthly family expenditure. The multivariate results from Models 1-4 (Table V and VI) for online shopping use indicated that participants with cell phone use, computer use, social media use, and mobile money transfer use were significantly more likely to use online shopping $(\mathrm{OR}=3.4, \mathrm{P}<$ $0.001 ; \mathrm{OR}=6.2, \mathrm{P}<0.001 ; \mathrm{OR}=3.8, \mathrm{P}<0.001$ AND $\mathrm{OR}=2.1$, $\mathrm{P}=0.037$, respectively) after adjusting for age, gender, employment status, education, and monthly family expenditure. The results of Model 2 for computer use indicated that the computer user are 6.2 times more likely to use online shopping compared to the non-user of computer $(\mathrm{OR}=6.22, \mathrm{P}$ $<0.001)$. The results of Model 3 for social media use indicated that social media user are 3.8 times more likely to use online shopping compared to the non-user of social media $(\mathrm{OR}=$ $3.78, \mathrm{P}<0.001)$. The results of Model 4 for mobile money transfer use indicated that the mobile money transfer user are 2.1 times more likely to use online shopping compared to the non-user of mobile money transfer service $(\mathrm{OR}=2.13, \mathrm{P}=$ $0.037)$.

TABLE V. AGE, GENDER, OCCUPATION, EDUCATION, AND FAMILY EXPENDITURE ADJUSTED ODDS RATIOS (ORS) AND 95\% CONFIDENCE INTERVALS (95\% CIS) FOR FACTORS ASSOCIATED WITH ONLINE SHOPPING USE (MODEL $1 \& 2$ )

\begin{tabular}{|c|c|c|c|c|c|c|c|}
\hline \multicolumn{5}{|c|}{ Model $1(\mathrm{~N}=512)$} & \multicolumn{3}{|c|}{ Model $2(\mathrm{~N}=512)$} \\
\hline & & OR & $\mathbf{P}$ & $95 \% \mathrm{CI}$ & OR & $\mathbf{P}$ & $95 \% \mathrm{CI}$ \\
\hline \multirow[b]{2}{*}{ Age group } & $15-29$ & Ref & & & & & \\
\hline & $\geq 30$ & 0.21 & $<0.001$ & $\begin{array}{l}0.09- \\
0.53\end{array}$ & 0.31 & 0.012 & $\begin{array}{l}0.12- \\
0.77 \\
\end{array}$ \\
\hline \multirow[b]{2}{*}{ Gender } & Female & Ref & & & & & \\
\hline & Male & 1.17 & 0.761 & $\begin{array}{l}0.39- \\
3.34\end{array}$ & 1.10 & 0.858 & $\begin{array}{l}0.37- \\
3.11\end{array}$ \\
\hline \multirow{2}{*}{$\begin{array}{l}\text { Employment } \\
\text { status }\end{array}$} & $\begin{array}{l}\text { Un } \\
\text { employed }\end{array}$ & Ref & & & & & \\
\hline & Employed & 3.59 & 0.016 & $\begin{array}{l}1.27- \\
10.26\end{array}$ & 2.43 & 0.093 & $\begin{array}{l}0.86- \\
6.91\end{array}$ \\
\hline \multirow{2}{*}{ Education } & $\begin{array}{l}\leq \text { Grade } \\
12\end{array}$ & Ref & & & & & \\
\hline & $\begin{array}{l}>\text { Grade } \\
12\end{array}$ & 3.34 & 0.001 & $\begin{array}{l}1.57- \\
6.89 \\
\end{array}$ & 2.43 & 0.020 & $\begin{array}{l}1.12- \\
5.10\end{array}$ \\
\hline \multirow{2}{*}{$\begin{array}{l}\text { Monthly } \\
\text { family } \\
\text { exp. }\end{array}$} & $\leq 10000$ & Ref & & & & & \\
\hline & $>10000$ & 1.43 & 0.357 & $\begin{array}{l}0.68- \\
3.13\end{array}$ & 1.44 & 0.347 & $\begin{array}{l}0.69- \\
3.15\end{array}$ \\
\hline \multirow{2}{*}{$\begin{array}{l}\text { Cell phone } \\
\text { user per } \\
\text { family }\end{array}$} & $\mathrm{n} \leq 2$ & Ref & & & & & \\
\hline & $\mathrm{n} \geq 3$ & 3.41 & $<0.001$ & $\begin{array}{l}1.76- \\
6.79 \\
\end{array}$ & & & \\
\hline \multirow{2}{*}{$\begin{array}{l}\text { Computer } \\
\text { use }\end{array}$} & No & & & & Ref & & \\
\hline & Yes & & & & 6.22 & $<0.001$ & $\begin{array}{l}3.14- \\
12.95 \\
\end{array}$ \\
\hline
\end{tabular}

With regard to the age groups, Models 1-4 showed that the participants whose age were 30 or older were significantly less likely to use online shopping compared to those who were aged between 15 and 29 years $(\mathrm{OR}=0.2, \mathrm{P}<0.001 ; \mathrm{OR}=0.3, \mathrm{P}=$ $0.012 ; \mathrm{OR}=0.2, \mathrm{P}<0.001 ; \mathrm{OR}=0.2, \mathrm{P}=0.002$, respectively). With regard to the employment status, Models 1, 3 and 4 showed a statistically significant relationship. The respondents who were employed were more likely to use online shopping compared to the respondents who were unemployed $(\mathrm{OR}=3.6$, $\mathrm{P}=0.016 ; \mathrm{OR}=3.2, \mathrm{P}=0.024 ; \mathrm{OR}=3.0, \mathrm{P}=0.030$, respectively). The employment status in Model 2 showed a border line relationship with online shopping use $(\mathrm{OR}=2.4, \mathrm{P}$ $=0.093$ ). With regard to the education, Models 1-4 showed a statistically significant relationship. The respondents who had higher education (more than 12 years of education) were more likely to use online shopping compared to the respondents who had the education level high school or less $(\mathrm{OR}=3.3, \mathrm{P}=$ $0.001 ; \mathrm{OR}=2.4, \mathrm{P}=0.020 ; \mathrm{OR}=3.1, \mathrm{P}=0.002 ; \mathrm{OR}=3.4, \mathrm{P}$ $<0.001$, respectively). However, with regard to monthly family expenditure, Models 1-4 did not show statistically significant relationship with online shopping use after adjusting for age, gender, employment status, education, and monthly family expenditure.

TABLE VI. AGE, GENDER, OCCUPATION, EDUCATION, AND FAMILY EXPENDITURE ADJUSTED ODDS RATIOS (ORS) AND 95\% CONFIDENCE INTERVALS (95\% CIS) FOR FACTORS ASSOCIATED WITH ONLINE SHOPPING USE (MODEL 3 \& 4)

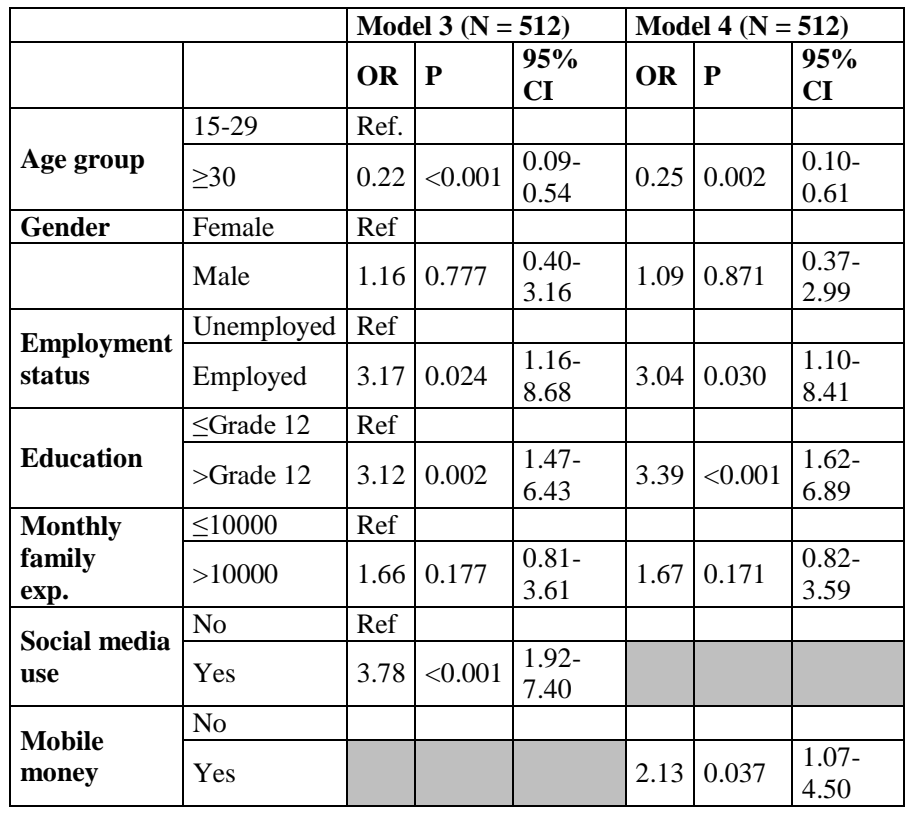

\section{DISCUSSION}

This is the first study to investigate the relationships and factors associated with online shopping in the western region of rural Bangladesh as per the authors' knowledge. This study found 51 respondents $(9 \%)$ out of 587 who had online shopping experience. The key finding was that online shopping use at the BoP community significantly associated with cell phone use, computer use, social media use, and mobile money transfer use after adjusting age, gender, employment status, education, and monthly family expenditure. Among them, computer use was the most significantly associated. Monthly family expenditure had a significant relationship before adjusting the variables. However, after adjusting with age, gender, employment status and education, it did not show significant relationship in all the four models in the analysis. This means, younger participants whose employment status was as employed and who had higher education showed a combined association with family expenditure to go for the 
decision of online shopping. Moreover, this research has found that, younger population (15-29 years old) were more likely to use online shopping compared to elderly population (more than 30 years old) at the BoP community. However, age group was not significant before adjusting the variables. The findings showed a similar trend in a study on global online shopping use trend where younger shoppers (21-34 years old) were the majority group [16]. The research did not find any significant relationship of online shopping with gender. However, findings on online shopping user status in Bangladesh showed that male were more likely to use online shopping compared to female [5]. The respondents who had higher education were more likely to use online shopping compared to the respondents who had the education of 12 years or less. The finding supports the online shopping use trend in developing countries [17]. This research has found a significant difference in the behavior of low family expenditure group and high family expenditure group for certain independent variables before adjusting with control variables. Education was significantly associated in high family expenditure group, but not in low family expenditure group. Similarly, social media use and mobile money transfer use were significantly associated with online shopping use in high family expenditure group; however, they were not significantly associated in low family expenditure group. Other independent variables such as cell phone user per family and computer use were significantly associated for both low family expenditure group and high family expenditure group.

\section{CONCLUSION}

This study aimed to depict current online shopping user status and the factors associated with online shopping use in BoP community. From the literature survey it identified that online shopping has been getting popular especially in the top and middle of the economic pyramid. Bottom of the economic pyramid are lack behind to enjoy the benefit of online shopping. This research targeted to find out current online shopping user and associated factors believing online shopping could be applicable in reducing BoP penalty. It collected data from a BoP environment in rural Bangladesh and after analyzing the data it found current status and factors associated to online shopping. This research had limitations as it focused on socio-demographic and behavioral factors. As a future work this research aims to identify the psychological factors of online shopping in BoP community.

\section{ACKNOWLEDGMENT}

This research is supported by Toyota Motor Corporation. We acknowledge Global Communication Center, Grameen
Communications for the implementation support in Bangladesh.

\section{REFERENCES}

[1] T. F. Stafford, A. Turan, and M. S. Raisinghani, "International and cross-cultural influences on online shopping behavior", Journal of Global Information Technology Management, vol. 7, issue 2, pp. 70-87, September 2014.

[2] A. Hussain, "E-commerce and beyond: opportunities for developing country SMEs", http://tradeforum.org/article, December 2013.

[3] A. Ahmed, M. A. Rahman, and T. Ohsugi, "E-commerce for the unreached community", Proc. IADIS International Conference ICT, Society and Human Beings (ICT 2011). July 24-26, 2011, Rome, Italy.

[4] C. Park and Y. Kim, "Identifying key factors affecting consumer purchase behavior in an online shopping context", International Journal of Retail \& Distribution Management, vol. 31, issue 1, pp. 16-29, 2003.

[5] P. Chahal, "A study on the role of consumers gender and age on online shopping", International Journal in Commerce, IT \& Social Sciences, vol. 2, issue 7, pp. 33-41, July 2015.

[6] S. Kurnia, J. Choudrie, R. M. Mahbubur, and B. Alzougool, "Ecommerce technology adoption: A Malaysian grocery SME retail sector study," Journal of Business Research, vol. 68, pp. 1906-1918, 2015.

[7] N. Kshetri, "Barriers to e-commerce and competitive business models in developing countries: A case study", Journal of Electronic Commerce Research and Applications, vol. 6, pp. 443-452, 2007.

[8] S. M. Forsythe and B. Shi, "Consumer patronage and risk perceptions in Internet shopping", Journal of Business Research, vol. 56, issue 11, pp. 867-875, November 2003.

[9] C. K. Prahalad, Fortune at the Bottom of the Pyramid, New Jersey: Pearson Publishing, 2005.

[10] T. London, R. Anupindi, and S. Sheth, "Lessons learned from ventures serving base of the pyramid producers", Journal of Business Research, vol. 63, pp. 582-594, 2010.

[11] A. Hammond, W. J. Kramer, J. Tran, R. Katz, and C. Walker, "The Next 4 Billion: Market Size and Business Strategy at the Base of the Pyramid", World Resources Institute Publications, 2007.

[12] M. T. Islam, M. A. Jafar, and A. R. Khan, "Factors motivating customers moving to online shops in Bangladesh", Bangladesh Journal of MIS, vol. 6, issue 2, pp. 51-61, June 2014.

[13] J. Suhan, "Acceptance of online shopping in Bangladesh: consumer's perspective", Journal of Business and Management, vol. 12, Issue 1, pp. 14-24, January 2015.

[14] C. Yoon, "The effects of national culture values on consumer acceptance of e-commerce: Online shoppers in China", Information and Management, vol. 46, Issue 5, pp. 294-301, June 2009.

[15] J. Kamau, A. Reberio-Hargrave, H. Saito, E. Abdullah, H. Okajima, and A. Ahmed, "Social Services on Wheels: A sustainable model to improve access in unreached communitites", IST-Africa proceedings, International Information Management Corporation IIMC, 2014.

[16] E-commerce: evolution or revolution in the fast-moving consumer goods world? Nielson global e-commerce report, August 2014.

[17] R. Rahayu and J. Day, "Determinant Factors of E-commerce Adoption by SMEs in Developing Country: Evidence from Indonesia", World Conference on Technology, Innovation and Entrepreneurship. Procedia Social and Behavioral Sciences, vol. 195, pp. $142-150,2015$. 